\title{
AN ACCRETING WHITE DWARF NEAR THE CHANDRASEKHAR LIMIT IN THE ANDROMEDA GALAXY
}

\author{
Sumin Tang ${ }^{1,2}$, Lars Bildsten ${ }^{1,3}$, William M. Wolf ${ }^{3}$, K. L. Li ${ }^{4}$, Albert K. H. Kong $^{4}$, Yi CaO ${ }^{2}$, S. Bradley Cenko $^{5,6}$, \\ Annalisa De Cia ${ }^{7}$, Mansi M. Kasliwal ${ }^{8,13}$, Shrinivas R. KulKarni ${ }^{2}$, Russ R. Laher ${ }^{9}$, Frank Masci $^{10}$, \\ Peter E. Nugent ${ }^{11,12}$, Daniel A. Perley ${ }^{2,13}$, Thomas A. Prince ${ }^{2}$, and Jason Surace ${ }^{9}$ \\ ${ }^{1}$ Kavli Institute for Theoretical Physics, University of California, Santa Barbara, CA 93106, USA \\ ${ }^{2}$ Division of Physics, Mathematics, and Astronomy, California Institute of Technology, Pasadena, CA 91125, USA \\ ${ }^{3}$ Department of Physics, University of California, Santa Barbara, CA 93106, USA \\ ${ }^{4}$ Institute of Astronomy and Department of Physics, National Tsing Hua University, Hsinchu 30013, Taiwan \\ ${ }^{5}$ Astrophysics Science Division, NASA Goddard Space Flight Center, Mail Code 661, Greenbelt, MD 20771, USA \\ ${ }^{6}$ Joint Space Science Institute, University of Maryland, College Park, MD 20742, USA \\ ${ }^{7}$ Department of Particle Physics and Astrophysics, Weizmann Institute of Science, Rehovot 76100, Israel \\ ${ }^{8}$ The Observatories, Carnegie Institution for Science, 813 Santa Barbara Street, Pasadena, CA 91101, USA \\ ${ }^{9}$ Spitzer Science Center, California Institute of Technology, M/S 314-6, Pasadena, CA 91125, USA \\ ${ }^{10}$ Infrared Processing and Analysis Center, California Institute of Technology, Pasadena, CA 91125, USA \\ ${ }^{11}$ Computational Cosmology Center, Lawrence Berkeley National Laboratory, 1 Cyclotron Road, Berkeley, CA 94720, USA \\ ${ }^{12}$ Department of Astronomy, University of California, Berkeley, CA 94720-3411, USA \\ Received 2014 January 10; accepted 2014 March 11; published 2014 April 16
}

\begin{abstract}
The intermediate Palomar Transient Factory (iPTF) detection of the most recent outburst of the recurrent nova (RN) system RX J0045.4+4154 in the Andromeda galaxy has enabled the unprecedented study of a massive $\left(M>1.3 M_{\odot}\right)$ accreting white dwarf (WD). We detected this nova as part of the near-daily iPTF monitoring of M31 to a depth of $R \approx 21 \mathrm{mag}$ and triggered optical photometry, spectroscopy and soft X-ray monitoring of the outburst. Peaking at an absolute magnitude of $M_{R}=-6.6 \mathrm{mag}$, and with a decay time of 1 mag per day, it is a faint and very fast nova. It shows optical emission lines of $\mathrm{He} / \mathrm{N}$ and expansion velocities of $1900-2600 \mathrm{~km} \mathrm{~s}^{-1}$ 1-4 days after the optical peak. The Swift monitoring of the X-ray evolution revealed a supersoft source (SSS) with $k T_{\text {eff }} \approx 90-110 \mathrm{eV}$ that appeared within 5 days after the optical peak, and lasted only 12 days. Most remarkably, this is not the first event from this system, rather it is an RN with a time between outbursts of approximately 1 yr, the shortest known. Recurrent X-ray emission from this binary was detected by ROSAT in 1992 and 1993, and the source was well characterized as a $M>1.3 M_{\odot}$ WD SSS. Based on the observed recurrence time between different outbursts, the duration and effective temperature of the SS phase, MESA models of accreting WDs allow us to constrain the accretion rate to $\dot{M}>1.7 \times 10^{-7} M_{\odot} \mathrm{yr}^{-1}$ and WD mass $>1.30 M_{\odot}$. If the WD keeps $30 \%$ of the accreted material, it will take less than a Myr to reach core densities high enough for carbon ignition (if made of $\mathrm{C} / \mathrm{O}$ ) or electron capture (if made of $\mathrm{O} / \mathrm{Ne}$ ) to end the binary evolution.
\end{abstract}

Key words: galaxies: individual (M31) - novae, cataclysmic variables - supernovae: general - white dwarfs $\mathrm{X}$-rays: binaries

Online-only material: color figures

\section{INTRODUCTION}

Classical novae are the observable outcome of unstable thermonuclear burning on accreting white dwarfs (WDs). In most tight binaries with WDs of typical masses of $M=0.8 M_{\odot}$, the recurrence time is tens of thousands of years. However, if the accretion rate, $\dot{M}$, is high and the WD mass is large, the time between flashes can become short enough so that the recurrence can be measured. Due to the large accretion rate and insignificant ejection mass loss, recurrent novae (RNe) have been proposed to grow toward the Chandrasekhar limit and could be promising progenitors of Type Ia supernovae (Starrfield et al. 1988; Di Stefano 2010). Within our galaxy, these RNe have, at the minimum, a recurrence time of $10 \mathrm{yr}$ (Schaefer 2010).

As the nearest large galaxy neighbor of the Milky Way, the Andromeda galaxy (M31) provides the best opportunity for studies of classical and RNe. Extensive photometric and spectroscopic surveys have been conducted to search for novae in M31, and have resulted in the discovery of over 900 novae, with over 100 having well-sampled light curves, optical spectra,

\footnotetext{
${ }^{13}$ Hubble Fellow.
}

or X-ray observations (Hubble 1929; Arp 1956; Darnley et al. 2004; Henze et al. 2010; Shafter et al. 2011; Cao et al. 2012; see also the web site maintained by Pietsch ${ }^{14}$ and references therein). There are six confirmed RNe in M31, and a few other strong candidates (Shafter et al. 2013).

During our nightly monitoring of M31 in the intermediate Palomar Transient Factory (iPTF; hereafter called simply PTF; Law et al. 2009), we discovered a transient at the location of a known nova in M31 (Tang et al. 2013), and confirmed it to be a RN with a recurrence time of $1 \mathrm{yr}$ with four optical outbursts detected in PTF from 2009 to 2013. During the optical novae, only a fraction of envelope is ejected (Starrfield et al. 1974), while the remaining envelope is expected to continue hydrogen burning. As the ejected envelope expands, the ejecta becomes optically thin, and a supersoft source (SSS) powered by hydrogen burning is expected to emerge after the optical nova (Sala \& Hernanz 2005; Wolf et al. 2013). Recent observational work has now made it clear that all novae have an extended supersoft phase whose duration and temperature depend solely on the WD Mass (Pietsch et al. 2005; Orio et al. 2010; Henze

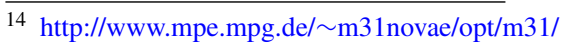




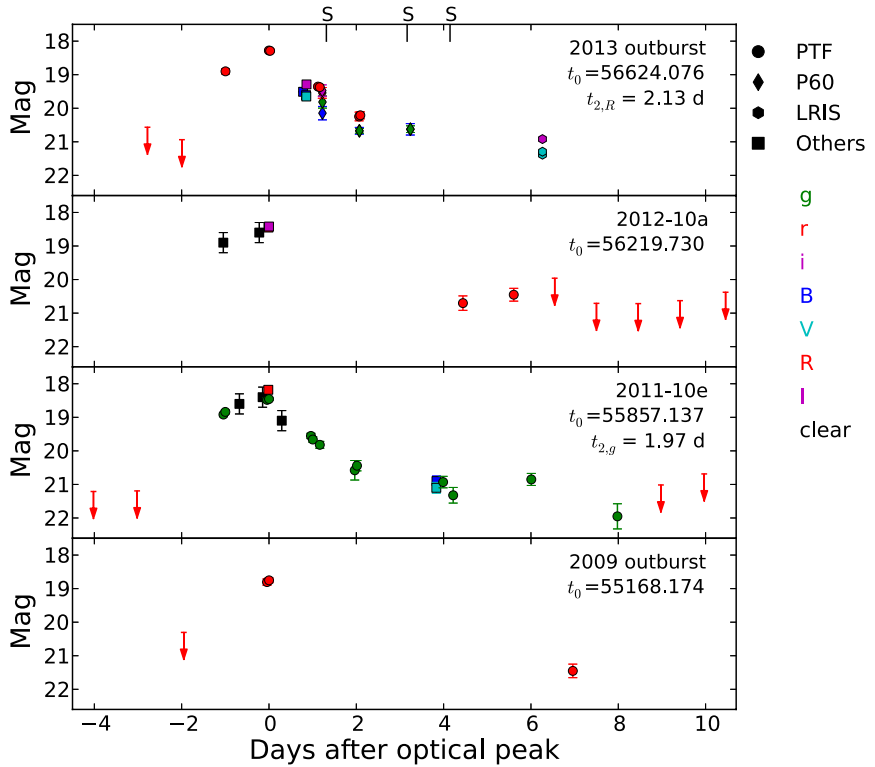

Figure 1. Optical light curve of RX J0045.4+4154. Four outbursts were detected in PTF from 2009 to 2013. PTF data are shown with circles (green for $g^{\prime}$ band and red for $R$ band). P60 follow-ups are shown with diamonds (blue for $B$ band, green for $g^{\prime}$ band, red for $r^{\prime}$ band, and magenta for $i^{\prime}$ band). LRIS follow-ups are shown with hexagons (cyan for $V$ band and magenta for $I$ band). Published photometry by others (Korotkiy \& Elenin 2010; Barsukova et al. 2011; Nishiyama \& Kabashima 2012; Shafter et al. 2012; Williams et al. 2013) is shown with squares (black for clear, blue for $B$ band, cyan for $V$ band, red for $R$ band, and magenta for $I / i^{\prime}$ band). Red arrows are $5 \sigma$ upper limits from PTF $R$-band images. On the top axis, the epochs of spectroscopic follow-up of the 2013 outburst are indicated by "S."

(A color version of this figure is available in the online journal.)

et al. 2014a). We show here that this apparent transient behavior is an excellent match to the supersoft phase of an $\mathrm{RN}$, and that the optical novae must have been missed in 1992, 1993 and 2001 when X-ray outbursts were seen (White et al. 1995; Williams et al. 2004). We describe the PTF discovery and optical follow-up observations in Section 2. Archival optical and X-ray studies are presented in Sections 3 and 4, respectively. Swift observations and analyses are presented in Section 5. Theoretical modeling is presented in Section 6. Our conclusion is in Section 7.

\section{PTF DISCOVERY AND OPTICAL FOLLOW-UP OBSERVATIONS}

On 2013 November $27.08 \mathrm{UT}^{15}{ }^{15}$ we detected a transient at $\alpha=00^{\mathrm{h}} 45^{\mathrm{m}} 28.89, \delta=41^{\circ} 54^{\prime} 10^{\prime \prime} .2$ with $R=18.9 \mathrm{mag}$ (Tang et al. 2013) in the nightly monitoring of M31 in the PTF using the 48 inch telescope at Palomar. It brightened to $R=18.3$ mag on November 28.08. No source was detected at the same location to $R<21 \mathrm{mag}$ in PTF images taken on November 26.08 and November 25.29. There was no detection in 270 nightly PTF $R$-band images taken between 2013 May 19 to November 13 to a similar depth during non-bright time. The transient is coincident within measurement uncertainties with the reported positions of three optical novae or novae candidates, i.e., He/N nova M31N2012-10a (Nishiyama \& Kabashima 2012; Shafter et al. 2012), nova candidates M31N2011-10e (Korotkiy \& Elenin 2010; Barsukova et al. 2011) and M31N 2008-12a (Nishiyama \& Kabashima 2008). It is also coincident with the position of a ROSAT recurrent supersoft transient RX J0045.4+4154 (White

\footnotetext{
15 All times are in UT.
}

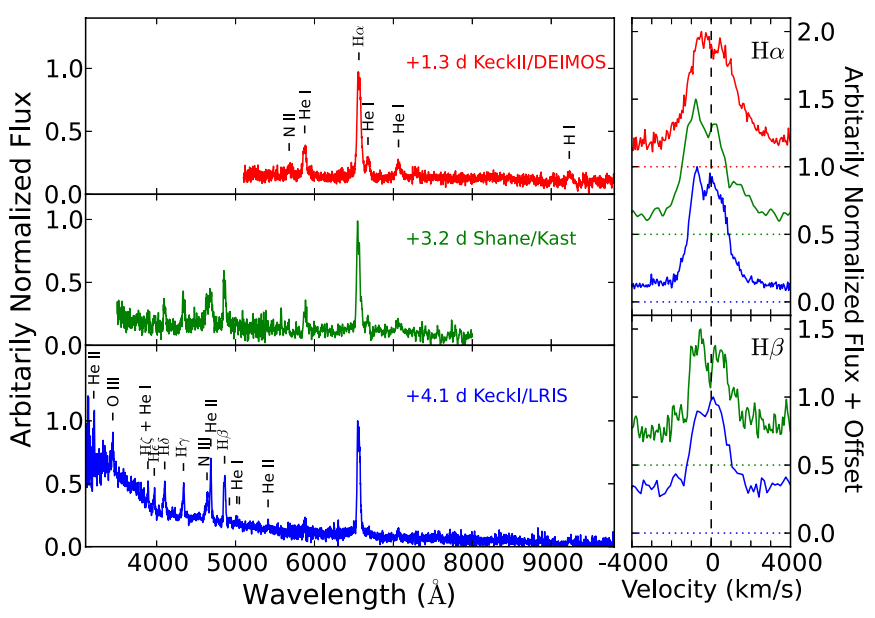

Figure 2. Optical spectra of RX J0045.4+4154 during the 2013 outburst. Left panels: arbitrarily normalized spectra. Each spectrum is labeled with the observation date, the telescope, and the instrument. Right panels: $\mathrm{H} \alpha$ and $\mathrm{H} \beta$ line profiles. The Kast spectrum is offseted by +0.5 , and the DEIMOS spectrum is offseted by +1.0 . The zero levels of the flux are shown in horizontal dotted lines. The position of zero velocity is marked by the vertical dashed line.

(A color version of this figure is available in the online journal.)

et al. 1995), which was the first discovery of outbursts from this source. Therefore, we refer to the transient as RX J0045.4+4154 hereafter.

Following our discovery, we initiated rapid photometric and spectroscopic follow-up observations. We obtained $B g^{\prime} r^{\prime} i^{\prime}$ observations on the Palomar 60 inch telescope (P60; Cenko et al. 2006) on November 29, November 30, and December 1, and VI images on the Low Resolution Imaging Spectrometer (LRIS; Oke et al. 1995) mounted on the Keck I 10 m telescope on December 4. Final reduction of the PTF images was performed using a forced-position point-spread function (PSF) photometry pipeline (Ofek et al. 2012; F. Masci et al. in preparation). The P60 and LRIS images were reduced using aperture photometry. We calibrated the LRIS images using the Local Group Survey catalog (Massey et al. 2006), and obtained a refined position for the transient of $\alpha=00^{\mathrm{h}} 45^{\mathrm{m}} 28^{\mathrm{s}} .847, \delta=41^{\circ} 54^{\prime} 10^{\prime} .05$ with uncertainty of 0 !' 1 (dominated by systematic uncertainties; the scattering of positions on the three LRIS images are $\approx 00^{\prime} 01$ ). The optical light curve in 2013 is shown in the top panel in Figure 1.

Optical spectroscopic follow-up of RX J0045.4+4154 was undertaken with the Deep Imaging Multi-Object Spectrograph (DEIMOS; Faber et al. 2003) mounted on the Keck II $10 \mathrm{~m}$ telescope on 2013 November 29 (1.3 days post peak), the Kast double spectrograph (Miller \& Stone 1993) on the Shane $3 \mathrm{~m}$ telescope at Lick Observatory on December 1 (3.2 days post peak), and LRIS mounted on the Keck I $10 \mathrm{~m}$ telescope on December 2 (4.1 days post peak). The spectral resolution is $4.2 \AA, 6 \AA$ (blue side) to $11 \AA$ (red side), and $7 \AA$, for the DEIMOS, Kast, and LRIS spectrum, respectively. Spectra were reduced with standard IRAF routines.

The spectroscopic series of RX J0045.4+4154 is shown in Figure 2. It showed strong emission lines of Balmer series, He I, He II, and N III. The observed optical lines place it into the "He/N" class of Williams (1992). The spectra are similar to the HET spectrum taken 0.6 days post peak of the 2012 outburst (M31N2012-10a; Shafter et al. 2012). Such similarity suggests that RN outbursts only depend on system parameters like the WD mass and binary properties. Compared with the 
Table 1

List of Observed Outbursts from RX J0045.4+4154

\begin{tabular}{|c|c|c|c|c|}
\hline $\begin{array}{l}t_{0, o p t^{\mathrm{a}}} \\
(\mathrm{UT})\end{array}$ & $\begin{array}{l}t_{0, X} \mathrm{~b} \\
(\mathrm{UT})\end{array}$ & $\begin{array}{l}\text { Time since Last Observed Outburst } \\
\text { (days) }\end{array}$ & Source & Reference \\
\hline & 2013 Dec 05 & & X-ray (Swift) & This paper; Henze et al. (2014b) \\
\hline 2013 Nov 28 & & 404 & Optical (PTF) & Tang et al. (2013); this paper \\
\hline 2012 Oct 19 & & 363 & Optical & Nishiyama \& Kabashima (2012); Shafter et al. (2012) \\
\hline 2011 Oct 23 & & 689 & Optical & Korotkiy \& Elenin (2010); Barsukova et al. (2011); this paper \\
\hline 2009 Dec 03 & & 342 & Optical (PTF) & This paper \\
\hline \multirow[t]{4}{*}{2008 Dec 26} & & & Optical & Nishiyama \& Kabashima (2008) \\
\hline & 2001 Sep 08 & & X-ray (Chandra) & Williams et al. (2004) \\
\hline & 1993 Jan 11 & 341 & $\mathrm{X}$-ray $(R O S A T)$ & White et al. (1995) \\
\hline & 1992 Feb 05 & & $\mathrm{X}$-ray $(R O S A T)$ & White et al. (1995) \\
\hline
\end{tabular}

Notes.

a Time of the optical peak.

b Time of the X-ray peak.

DEIMOS spectrum, He I lines weakened significantly in the LRIS spectrum, suggesting a decrease in the shell ionization during this period. The FWHM of $\mathrm{H} \alpha$ emission line decreased, from $2600 \mathrm{~km} \mathrm{~s}^{-1}$ in the DEIMOS spectrum, to $1900 \mathrm{~km} \mathrm{~s}^{-1}$ in the LRIS spectrum, suggesting decreased ejecta velocity. Such velocity is at the low end of He/N nova (Shafter et al. 2011), and is at the low end of RNe (see, e.g., Kato \& Hachisu 2003; Yamanaka et al. 2010).

The galactic extinction along the line of sight of RX J0045.4+4154 is $A_{R}=0.134$ and $A_{g}=0.205$ (Schlafly \& Finkbeiner 2011), which is the lower limit of extinction of the object. To estimate the line-of-sight extinction contribution from M31 from the LRIS spectrum, we subtract the continuum, and measure the flux ratio of $\mathrm{H} \alpha / \mathrm{H} \beta$ to be 3.89. Assuming case B recombination (optically thick in $\operatorname{Ly} \alpha$ Lines, optically thin in all other Hydrogen lines), the extinction is $A_{R}=0.65 \pm 0.23$ and $A_{g}=1.0 \pm 0.35$ (uncertainty comes from the range in expected ratios for case B of 2.76-3.30; Osterbrock \& Ferland 2006), which corresponds to $N_{\mathrm{H}}=1.5 \pm 0.5 \times 10^{21} \mathrm{~cm}^{-2}$ (Predehl \& Schmitt 1995). If the nebula is not optically thin in Balmer lines or if the collisional excitation is non-negligible, a higher $\mathrm{H} \alpha / \mathrm{H} \beta$ ratio is expected relative to the case $\mathrm{B}$ recombination, leading to an overestimated extinction. Therefore, the above dust extinction and hydrogen column density should be regarded as upper bounds.

\section{ARCHIVAL OPTICAL OBSERVATIONS}

\subsection{Past Outbursts}

Archival work within PTF revealed other outbursts. We confirmed outbursts in 2012 October (He/N nova M31N201210a; Nishiyama \& Kabashima 2012; Shafter et al. 2012) and 2011 October (nova candidate M31N2011-10e; Korotkiy \& Elenin 2010; Barsukova et al. 2011), and identified another un-reported outburst in 2009 December. The transient is also coincident with the reported positions of nova candidate M31N 2008-12a (Nishiyama \& Kabashima 2008). We also went back to Arp's survey (1956) of M31 novae in the 1950s and found nothing at this location in his catalog.

The observed outbursts are listed in Table 1. The recurrence time is 341 days from ROSAT (White et al. 1995), and the recurrence times between the five most recent outbursts (2008-2013) are 342, 689, 363, and 404 days, respectively. As- suming a recurrence time between 330-410 days, we expect an outburst in 2010 during October 29 to November 27. During this time, there are 30 PTF images ( $R$ band, typical limiting mag 20.5-21 mag), 6 images taken by P60 ( $g^{\prime}$ and $r^{\prime}$ bands, typical limiting mag 21-22 mag), and 6 images taken by MegaCam on the Canada-France-Hawaii Telescope (CFHT; Boulade et al. 2003) on 2010 October 31 in $u^{\prime}, g^{\prime}$, and $r^{\prime}$ with 200-600 s exposures (limiting mag $\approx 24 \mathrm{mag}$ ). No outburst was detected. If there is an outburst with light curve similar to the 2013 and 2011 novae, it will be detected by PTF during $t-t_{0}=-1$ to 1 (where $t_{0}$ is the optical peak time), by P60 during $t-t_{0}=-1$ to 1 , and by CFHT/MegaCam during $t-t_{0}=-1$ to 7 . Only two possible windows are left if there was a nova in 2010 , i.e., November 10-12, or November 20-27.

The optical light curves of the 2009, 2011, 2012, and 2013 outbursts are shown in Figure 1, and are similar. We have daily sampled $g^{\prime}$-, and $R$-band light curves covering the rise, peak, and decay of the 2011 and 2013 outbursts. The derived $t_{2}$ (time to decay from the peak by $2 \mathrm{mag}$ ) is $t_{2, g}=2.0$ days in the 2011 outburst, and $t_{2, R}=2.1$ days in the 2013 outburst, making it among the fastest novae (Cao et al. 2012). The declines within 2 mag from the peak are more or less linear, and thus linear regression is used to derive $t_{2}$. The peak magnitude in the 2013 outburst is $R=18.34 \pm 0.08 \mathrm{mag}$ at $\mathrm{MJD}=56624.076$ from PTF data. The peak magnitudes in the 2011 outburst is $g=18.51 \pm 0.09 \mathrm{mag}$ at $\mathrm{MJD}=55857.137$ from PTF data, and $R=18.18 \pm 0.08 \mathrm{mag}$ at $\mathrm{MJD}=55857.121$ from Barsukova et al. (2011).

The simultaneous or nearly simultaneous photometric measurements in the 2011 and 2013 outbursts are listed in Table 2. Assuming an intrinsic effective temperature of $T \approx 8200 \mathrm{~K}$ as observed in typical novae, the inferred extinction is $A_{R} \approx 0-0.7$ $\left(N_{\mathrm{H}}<1.6 \times 10^{21} \mathrm{~cm}^{-2}\right)$.

Adopting an extinction of $A_{R}=0.45$ and $A_{g}=0.7$ $\left(N_{\mathrm{H}}=1.0 \times 10^{21} \mathrm{~cm}^{-2}\right)$, which is consistent with both the Balmer decrement and available colors of the outbursts, and given the distance modulus of M31 of 24.4 (Vilardell et al. 2010), the peak absolute magnitude of the 2013 and 2011 outbursts is then $M_{R}=-6.5 \mathrm{mag}$, and $M_{g}=-6.6 \mathrm{mag}$, respectively. With the fastest decline rate $\left(1 \mathrm{mag} \mathrm{d}^{-1}\right)$ yet faint peak magnitude among novae, RX J0045.4+4154 is an extreme outlier in the canonical maximum-magnitude-rate-of-decline relation (della Valle \& Livio 1995). As discussed by Kasliwal et al. (2011), such 
Table 2

Optical Photometry of RX J0045.4+4154 during the 2013 and 2011 Outbursts with (Nearly) Simultaneous Multi-band Observations

\begin{tabular}{|c|c|c|c|c|c|c|c|c|c|}
\hline MJD & $\begin{array}{l}t-t_{0} \\
\text { (days) }\end{array}$ & $\begin{array}{c}B \\
(\mathrm{mag})\end{array}$ & $\begin{array}{c}V \\
(\mathrm{mag})\end{array}$ & $\begin{array}{c}R \\
\text { (mag) }\end{array}$ & $\begin{array}{c}I \\
(\mathrm{mag})\end{array}$ & $\begin{array}{c}g^{\prime} \\
(\mathrm{mag})\end{array}$ & $\begin{array}{c}r^{\prime} \\
(\mathrm{mag})\end{array}$ & $\begin{array}{c}i^{\prime} \\
(\mathrm{mag})\end{array}$ & Ref. $^{a}$ \\
\hline \multicolumn{10}{|c|}{$2013, t_{0}=56624.08$ in MJD } \\
\hline 56624.93 & 0.85 & $19.61 \pm 0.01$ & $19.65 \pm 0.02$ & & & & & $19.29 \pm 0.02$ & W13 \\
\hline 56625.26 & 1.18 & $20.15 \pm 0.20$ & & & & $19.82 \pm 0.18$ & $19.54 \pm 0.16$ & $19.47 \pm 0.17$ & P60 \\
\hline 56626.15 & 2.07 & $20.67 \pm 0.10$ & & $20.32 \pm 0.16$ & & $20.67 \pm 0.07$ & & & PTF and P60 \\
\hline 56627.32 & 3.24 & $20.63 \pm 0.17$ & & & & $20.62 \pm 0.10$ & & & P60 \\
\hline \multicolumn{10}{|c|}{$2011, t_{0}=55857.1$ in MJD } \\
\hline 55857.1 & 0 & & & $18.18 \pm 0.08$ & & $18.51 \pm 0.09$ & & & B11; PTF \\
\hline
\end{tabular}

Notes. ${ }^{a}$ References: W13 is Williams et al. 2013; B11 is Barsukova et al. 2011. Others are either PTF photometry or our follow-up using Palomar 60-inch or KeckI/LRIS.

faint and fast novae can arise from progenitors containing high accretion rate and relatively massive WDs (thus lower envelope masses at hydrogen ignition; see, e.g., Wolf et al. 2013), as we expect for RNe.

\subsection{Archival HST Observations}

Following the candidate progenitor reported in Williams et al. (2013), we re-analyzed the Hubble Space Telescope (HST) archival exposures to investigate photometric properties of RX J0045.4+4154 in quiescence. Besides the Advanced Camera for Surveys (ACS)/WFC data (filters: F475W and F814W; date: 2010 August 7) mentioned in the report, we made use of newer ACS/WFC (filters: F475W and F814W; date: 2012 January 10) and WFC3/UVIS and IR (filters: F110W, F160W, F275W, and F336W; date: 2011 January 25 and August 31) observations in the analysis. To search for the progenitor, accurate astrometry with precision down to 0.'02-0.'07 between our Keck/LRIS image (see Section 2) and the HST images were performed based on 5-18 bright reference stars. Within the Keck/LRIS error circle (astrometric uncertainty dominated) of RX J0045.4+4154, a clear source is present in the optical $/ U V$-band $H S T$ images but is undetectable in the $I R$ band. We measured magnitudes of the detected source through the Dolphot PSF photometry package (Dolphin 2000) with HST-dedicated parameters suggested in the manual. All calculated photometric measures are listed in Table 3. The mean magnitudes for the optical/UV filters are $\mathrm{F} 275 \mathrm{~W}=23.07, \mathrm{~F} 336 \mathrm{~W}=22.96, \mathrm{~F} 475 \mathrm{~W}=24.24$, and $\mathrm{F} 814 \mathrm{~W}=23.91 .^{16}$ For the $I R$ bands, we estimated the upper limits by examining the faintest stars detected by the $H S T$ exposures, which gives us F110W $>24.44$ (exposure: $800 \mathrm{~s}$ ) and $\mathrm{F} 160 \mathrm{~W}>25.22$ (exposure: $1700 \mathrm{~s}$ ). Variability is present in all optical $/ U V$ bands with amplitudes up to $0.3 \mathrm{mag}$ for F275W, 0.6 mag for F336W, 0.5 mag for F475W, and 0.2 mag for $\mathrm{F} 814 \mathrm{~W}$, likely from the high accretion rate disk. There is a significant change in color between 2010 and 2012 with $\Delta(B-I) \approx-0.3$ suggesting the system was possibly in a different phase of the nova recurrent cycle or/and at a different accretion rate. The results are consistent with an independent study on the same object by Darnley et al. (2014).

\section{ARCHIVAL X-RAY DATA}

In the M31 s ROSAT PSPC survey catalog (Supper et al. 2001), a recurrent supersoft X-ray transient RXJ 0045.4+4154,

\footnotetext{
16 See Table 3 for details.
}

identified by White et al. (1995), is spatially coincident with the optical RN (offset: 4"; uncertainty 13"). RXJ 0045.4+4154 showed two distinct supersoft X-ray outbursts of duration $\approx 5$ days in 1992 February and 1993 January (340 days apart) (see White et al. 1995, Figure 2), in which rapid luminosity changes over the 5 day intervals were clearly seen while a comparably bright X-ray source was completely absent before or after the outbursts. White et al. (1995) interpreted the supersoft X-rays as the consequence of thermonuclear burning of hydrogen on the surface of a WD and estimated the WD atmosphere temperature to be $k T_{\mathrm{bb}} \approx 90 \mathrm{eV}$ by fitting the spectrum with a blackbody model, inferring a peak luminosity (absorption corrected) of $L_{X} \approx 10^{38} \mathrm{erg} \mathrm{s}^{-1}(0.1-2 \mathrm{keV})$ and a blackbody radius of $R_{\mathrm{bb}} \approx 4.7 \times 10^{8} \mathrm{~cm}\left(D_{\mathrm{M} 31}=780 \mathrm{kpc}\right.$ assumed). From an $\mathrm{O}$ VIII ionization edge detection at $0.87 \mathrm{keV}$, RXJ 0045.4+4154 was believed to be a massive WD with $M>1.3 M_{\odot}$ (i.e., $\left.\log (g)>8.75\right)$.

Williams et al. (2004) reported a Chandra/HRC transient detection in 2001 September, in which the X-ray transient, named n1-85, was mostly quiescent $\left(L_{X} \leqslant 2 \times 10^{36} \mathrm{erg} \mathrm{s}^{-1}\right)$, but was detected at one epoch with $L_{X} \approx 6 \times 10^{37} \mathrm{erg} \mathrm{s}^{-1}$ (see Williams et al. 2004, Figure 6). Their reported coordinates of n1-85 is $9^{\prime \prime}$ offset from the optical position of RX J0045.4+4154, seemingly making an association with RX J0045.4+4154 unlikely. However, we registered the Chandra/HRC image to the Swift/ X-ray Telescope (XRT) WCS frame to investigate possible association. Using four common X-ray sources detected by both the instruments in the field, the astrometric corrected offset of n1-85 and RXJ 0045.4+4154 dropped to about $1^{\prime \prime}$, which is smaller than the positional uncertainty of the XRT position (1".6), hence, we are confident of the association.

\section{Swift OBSERVATIONS}

\subsection{Swift XRT and UVOT Light Curves}

Given the short X-ray outburst duration observed in ROSAT (White et al. 1995), we launched high cadence target of opportunity X-ray and UV observations with the Swift observatory (Gehrels et al. 2004). Another Swift campaign was carried out by M. Henze and collaborators (Henze et al. 2013a) following our optical discovery (Tang et al. 2013). RX J0045.4+4154 was observed with the Swift XRT (Burrows et al. 2005) and the Ultraviolet/Optical Telescope (UVOT; Roming et al. 2005) in a series of 36 observations beginning on 2013 December 3 (5 days after the optical peak). Typically 4-6 ks observations were taken in 1-2 ks snapshots each day from December 3 to December 16, 
Table 3

HST Observations of RX J0045.4+4154

\begin{tabular}{|c|c|c|c|c|c|}
\hline Filter ID & $\begin{array}{c}\text { Observing Time } \\
\text { (yyyy-mm-dd hh:mm:ss) }\end{array}$ & $\begin{array}{c}\text { Days after }^{\mathrm{a}} \\
\text { Previous Nova }\end{array}$ & $\begin{array}{l}\text { Days before }^{a} \\
\text { Next Nova }\end{array}$ & $\begin{array}{c}\text { Exposure } \\
\text { (s) }\end{array}$ & $\begin{array}{c}\text { Vega Magnitude } \\
\text { (mag) }\end{array}$ \\
\hline \multirow[t]{4}{*}{ F275W } & 2011 Jan 25 04:56:24 & $418(74)$ & 271 & 350 & $23.244 \pm 0.118$ \\
\hline & 2011 Jan 25 05:20:52 & $418(74)$ & 271 & 660 & $23.078 \pm 0.072$ \\
\hline & 2011 Aug 31 12:18:37 & $636(292)$ & 53 & 350 & $23.075 \pm 0.103$ \\
\hline & 2011 Aug 31 12:41:25 & $636(292)$ & 53 & 575 & $22.955 \pm 0.079$ \\
\hline \multirow[t]{4}{*}{ F336W } & 2011 Jan 25 04:44:43 & $418(74)$ & 271 & 550 & $23.309 \pm 0.054$ \\
\hline & 2011 Jan 25 05:04:54 & $418(74)$ & 271 & 800 & $23.118 \pm 0.040$ \\
\hline & 2011 Aug 31 12:06:56 & $636(292)$ & 53 & 550 & $22.715 \pm 0.042$ \\
\hline & 2011 Aug 31 12:27:07 & $636(292)$ & 53 & 700 & $22.738 \pm 0.037$ \\
\hline \multirow[t]{8}{*}{ F475W } & 2010 Aug 07 12:27:39 & 247 & $431(87)$ & 600 & $24.041 \pm 0.022$ \\
\hline & 2010 Aug 07 12:40:20 & 247 & $431(87)$ & 370 & $24.041 \pm 0.029$ \\
\hline & 2010 Aug 07 12:49:08 & 247 & $431(87)$ & 370 & $24.035 \pm 0.029$ \\
\hline & 2010 Aug 07 12:57:56 & 247 & $431(87)$ & 370 & $23.989 \pm 0.028$ \\
\hline & 2012 Jan 10 02:45:00 & 79 & 284 & 700 & $24.448 \pm 0.028$ \\
\hline & 2012 Jan 10 02:59:21 & 79 & 284 & 360 & $24.451 \pm 0.038$ \\
\hline & 2012 Jan 10 03:07:59 & 79 & 284 & 360 & $24.410 \pm 0.037$ \\
\hline & 2012 Jan 10 03:16:37 & 79 & 284 & 470 & $24.478 \pm 0.033$ \\
\hline \multirow[t]{6}{*}{ F814W } & 2010 Aug 07 10:44:08 & 247 & 431 & 350 & $23.846 \pm 0.045$ \\
\hline & 2010 Aug 07 10:52:38 & 247 & $431(87)$ & 700 & $23.871 \pm 0.031$ \\
\hline & 2010 Aug 07 11:06:56 & 247 & $431(87)$ & 455 & $23.815 \pm 0.037$ \\
\hline & 2012 Jan 10 00:23:51 & 79 & 284 & 350 & $23.981 \pm 0.049$ \\
\hline & 2012 Jan 10 01:09:03 & 79 & 284 & 800 & $23.967 \pm 0.039$ \\
\hline & 2012 Jan 10 01:25:01 & 79 & 284 & 550 & $23.964 \pm 0.038$ \\
\hline
\end{tabular}

Notes. ${ }^{a}$ Days in parenthesis are the ones assuming there was a missing nova on 2010 November 12 , which is the middle point between the 2009 and the 2011 novae.

and 7-8 ks observations once every 3 days from December 17 to December 23. The total exposure time is $97.6 \mathrm{ks}$.

A highly variable source is detected in both XRT and UVOT at the optical position of RX J0045.4+4154 (see also Henze et al. 2013b). The enhanced position given by the online Swift XRT pipeline software of the UK Swift Science Data Centre at the University of Leicester (Evans et al. 2007) is $\alpha=00^{\mathrm{h}} 45^{\mathrm{m}} 28^{\mathrm{s}} .80$, $\delta=41^{\circ} 54^{\prime} 08^{\prime \prime} .8$ with a $90 \%$ error of $1^{\prime \prime} .6$, which is consistent with the optical position of the nova. The Swift XRT light curve of RX J0045.4+4154 given by the same pipeline is shown in black in the top panel of Figure 3. The first detection in optical is 1 day before the optical peak, and we have a $5 \sigma$ upper limit of $R>21$ mag 2 days before the optical peak. Hence the onset of the thermonuclear runaway (TNR) is 1-2 days before the optical peak. Therefore, from the XRT light curve, we measured the turn-on time (defined as the time it takes for the source to emerge as a SSS after the onset of TNR) of $<6-7$ days, and turnoff time (defined as the time it takes for the source to disappear in X-rays after the onset of TNR) of 18-19 days. Both timescales are among the shortest ones measured for novae (Henze et al. 2014a).

The UVOT data were reduced with the HEASoft V6.15 package and with the calibration files released in 2013 January. Aperture photometry was performed using UVOTPRODUCT, with a $5^{\prime \prime}$ radius for the source, and an annulus with inner radius $27^{\prime \prime}$ and outer radius $35^{\prime \prime}$ for background, as recommended by Poole et al. (2008). No bright UV source is located in the background annulus. The resulting UVOT light curves are shown in the bottom panel of Figure 3. The magnitudes are in the Vega system. It showed significant short-time variations, notably 1 mag variations on hourly timescales in the uvw2 filter (1928 $\pm 657 \AA$; Poole et al. 2008) during the five snapshots in the first Swift observation. It also showed a relatively monotonical decline on longer timescales (a few days) after the first Swift

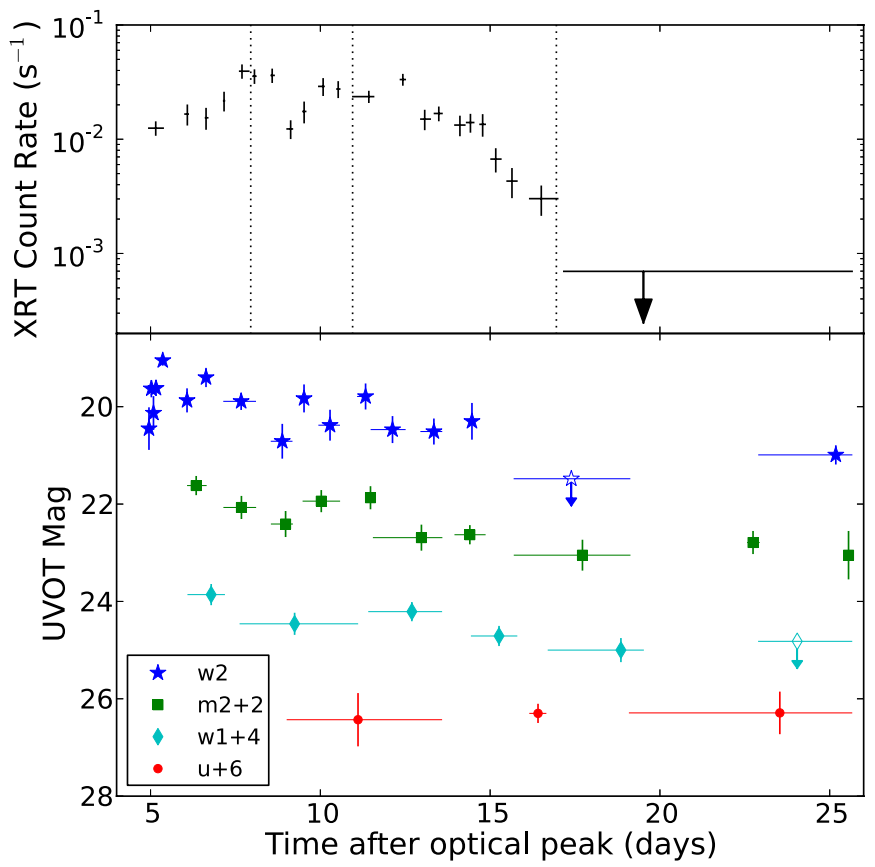

Figure 3. Top: Swift XRT light curve of RX J0045.4+4154. Arrows are $3 \sigma$ upper limits. Vertical dotted lines mark the time bins used in spectral analysis (Figure 4 and Table 4). Bottom: Swift UVOT light curves of RX J0045.4+4154. The uvm2, uvw1, and $u$-band light curves are shifted by constant values as indicated in the legend. Open symbols with arrows are $3 \sigma$ upper limits.

(A color version of this figure is available in the online journal.)

observation. The long-term variation amplitude during the Swift campaign is about $2 \mathrm{mag}, 1.4 \mathrm{mag}$ and $1 \mathrm{mag}$ in the uvw2, uvm2 $(2246 \pm 498 \AA)$ and uvw1 $(2600 \pm 693 \AA)$ filters, respectively. No significant variation is detected in the $u$ band $(3465 \pm 785 \AA$ ). 
Table 4

X-ray Spectral Fitting of RX J0045.4+4154

\begin{tabular}{|c|c|c|c|c|c|}
\hline $\begin{array}{l}\text { Time }^{\mathrm{a}} \\
\text { (days) }\end{array}$ & $\begin{array}{c}N_{\mathrm{H}} \\
\left(10^{21} \mathrm{~cm}^{-2}\right)\end{array}$ & $\begin{array}{c}\text { Temperature }\left(T_{\mathrm{bb}}\right)^{\mathrm{b}} \\
(\mathrm{eV})\end{array}$ & $\begin{array}{l}\operatorname{Radius}^{\mathrm{c}}\left(R_{\mathrm{bb}}\right) \\
\quad\left(10^{8} \mathrm{~cm}\right)\end{array}$ & $\begin{array}{l}\text { Blackbody Emission }^{\mathrm{d}}\left(L_{X}\right) \\
\quad\left(10^{38} \mathrm{erg} \mathrm{s}^{-1}\right)\end{array}$ & $\begin{array}{c}\chi_{v}^{2}(\mathrm{dof}) \\
\left(10^{38} \mathrm{erg} \mathrm{s}^{-1}\right)\end{array}$ \\
\hline \multicolumn{6}{|c|}{ Single Absorbed Blackbody with a Fixed $N_{\mathrm{H}}=1.0 \times 10^{21} \mathrm{~cm}^{-2}$} \\
\hline $5-8$ & & $94_{-6}^{+6}$ & $4.7_{-2.7}^{+3.3}$ & $1.2_{-0.3}^{+0.1}$ & \\
\hline $8-11$ & 1.0 (fixed) & $112_{-7}^{+7}$ & $2.9_{-1.6}^{+1.9}$ & $1.2_{-0.2}^{+0.1}$ & $1.40(42)$ \\
\hline $11-17$ & & $95_{-5}^{+5}$ & $3.4_{-1.9}^{+2.3}$ & $0.7_{-0.1}^{+0.1}$ & \\
\hline \multicolumn{6}{|c|}{ Single Absorbed Blackbody with a Fixed $N_{\mathrm{H}}=1.5 \times 10^{21} \mathrm{~cm}^{-2}$} \\
\hline $5-8$ & & $87_{-5}^{+5}$ & $7.3_{-4.3}^{+5.4}$ & $2.0_{-0.4}^{+0.2}$ & \\
\hline $8-11$ & 1.5 (fixed) & $104_{-6}^{+6}$ & $4.3_{-2.4}^{+2.9}$ & $1.8_{-0.3}^{+0.1}$ & $1.27(42)$ \\
\hline $11-17$ & & $88_{-5}^{+5}$ & $5.1_{-2.9}^{+3.5}$ & $1.1_{-0.2}^{+0.1}$ & \\
\hline
\end{tabular}

Notes.

a Time after the optical peak in 2013.

b All uncertainty ranges are $90 \%$ confidence interval.

c The radii of the blackbody were calculated using the best-fit normalizations (i.e., $\propto R_{\mathrm{bb}}^{2} / D_{\mathrm{M} 31}^{2}$ with $\left.D_{\mathrm{M} 31}=780 \mathrm{kpc}\right)$.

d The luminosities $(0.3-1 \mathrm{keV})$ are absorption corrected with an assumption of $D_{\mathrm{M} 31}=780 \mathrm{kpc}$ while the uncertainties were estimated by approximating $\Delta L_{\mathrm{unabs}} / L_{\mathrm{unabs}} \approx \Delta L_{\mathrm{obs}} / L_{\mathrm{obs}}$.

The relatively smaller variation amplitudes in UV compared with X-ray are consistent with the scenario of decreasing effective temperature in the late stage of SSS.

\subsection{Effective Temperature and Column Density Evolution}

We obtained the Swift data including the corresponding ancillary files from the Swift quick look data archive, extracted the level-2 event files to spectra using HEASoft version 6.14, and performed spectral fittings using XSPEC version 12.8.1 with the an absorbed blackbody model (i.e., phabs*bbobyrad). Given that the X-ray variability is high over the entire supersoft phase, we split the spectrum into parts according to the observing time to investigate the luminosity and temperature evolutions of the system. We divided the observations into three groups (Table 4) and fitted each with an absorbed blackbody. Due to a low photon count, we assumed two possible $N_{\mathrm{H}}$, i.e., $1.0 \times 10^{21} \mathrm{~cm}^{-2}$ and $1.5 \times 10^{21} \mathrm{~cm}^{-2}$, to increase the degrees of freedom of the fits. The resulting fits for $n_{H}=1.5 \times 10^{21} \mathrm{~cm}^{-2}$ are shown in Figure 4 . The assumed $N_{\mathrm{H}}$ are consistent with the extinction measured from the Balmer decrement $\left(N_{\mathrm{H}}<1.5 \times 10^{21} \mathrm{~cm}^{-2}\right)$ and optical colors $\left(N_{\mathrm{H}}=0-1.6 \times 10^{21} \mathrm{~cm}^{-2}\right)$, as well as the results from an independent study on the same object by Henze et al. (2014b) who estimated a $N_{\mathrm{H}}$ of $1.1-1.6 \times 10^{21} \mathrm{~cm}^{-2}$ from the Swift data.

As listed in Table 4, the fits show significant variabilities in temperature and luminosity and all best-fit parameters are consistent with the ROSAT outbursts. The limited number of XRT photons and our lack of knowledge of the metallicity at the stellar photosphere inhibited our use of the more accurate stellar atmosphere models of Rauch et al. (2010) that were constructed for hot and massive WDs.

\section{HIGH M AND $M$ NOVA MODELS}

The short nova recurrence time, rapid evolution as an X-ray source and high surface temperature during the SSS phase all consistently point to a high mass WD. To quantify just how large the WD mass must be to explain the observations, we undertook an expansion of the recent work of Wolf et al. (2013) using the Modules for Experiments in Astrophysics (MESA rev. 5596; Paxton et al. 2011, 2013). We simulated WDs with

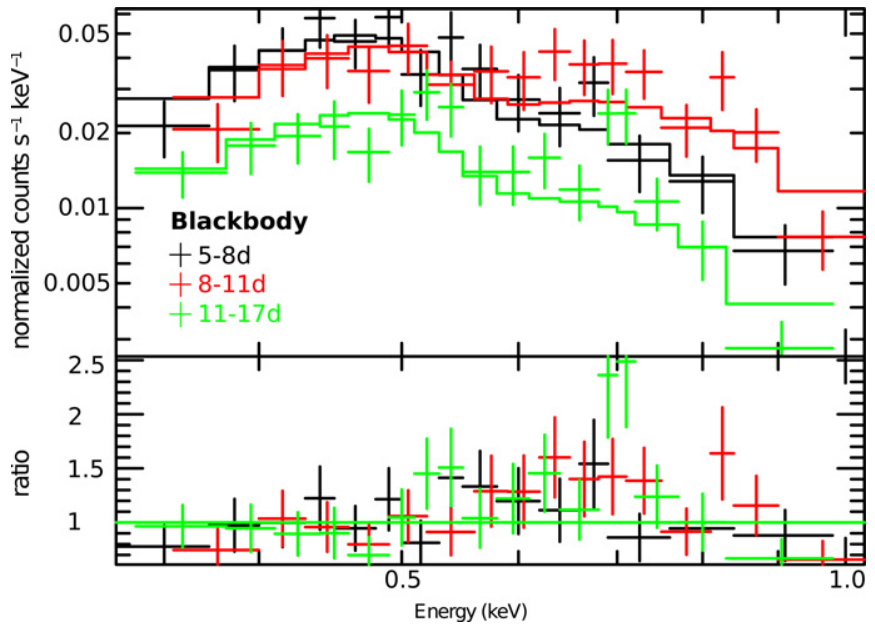

Figure 4. Swift/XRT spectra (from 0.3 to around $1 \mathrm{keV}$ ) of RX J0045.4+4154 taken at different time bins during the supersoft phase are shown with their best-fit black body spectral models assuming a fixed $N_{\mathrm{H}}=1.5 \times 10^{21} \mathrm{~cm}^{-2}$. Black for days 5-8 after the optical peak, red for days 8-11, and green for days 11-17.

(A color version of this figure is available in the online journal.)

$M=1.30,1.32,1.34$, and $1.36 M_{\odot}$ accreting material with solar composition, focusing on models that yielded the observed recurrence time of $1 \mathrm{yr}$, yielding an accretion rate range of $1.7 \times 10^{-7}<\dot{M} / M_{\odot} \mathrm{yr}^{-1}<3.3 \times 10^{-7}$. These model WDs have core temperatures of $T_{c}=3 \times 10^{7} \mathrm{~K}$, except for the $1.36 M_{\odot}$ model, which had $T_{c}=6 \times 10^{7} \mathrm{~K}$. The value of $T_{c}$ does not impact the outcome at these high $\dot{M}$ 's due to the heat buffer created by the even hotter helium layer $\left(T_{\mathrm{He}} \approx 10^{8} \mathrm{~K}\right)$ (Prialnik \& Kovetz 1995; Cassisi et al. 1998; Piersanti et al. 1999; Yaron et al. 2005; Wolf et al. 2013). The mass loss prescription during the novae events is a super Eddington wind, as described in Denissenkov et al. (2013) and Wolf et al. (2013).

These calculations immediately show that the WD in RX J0045.4+4154 has a mass of at least $1.3 M_{\odot}$, as lower mass WDs could not yield a minimum recurrence time as short as 1 yr. The $\dot{M}$ 's for the $1.32,1.34$, and $1.36 M_{\odot}$ models that yielded $t_{\text {recur }}=1 \mathrm{yr}$ were, respectively, $3.1 \times 10^{-7}, 2.1 \times 10^{-7}$, 


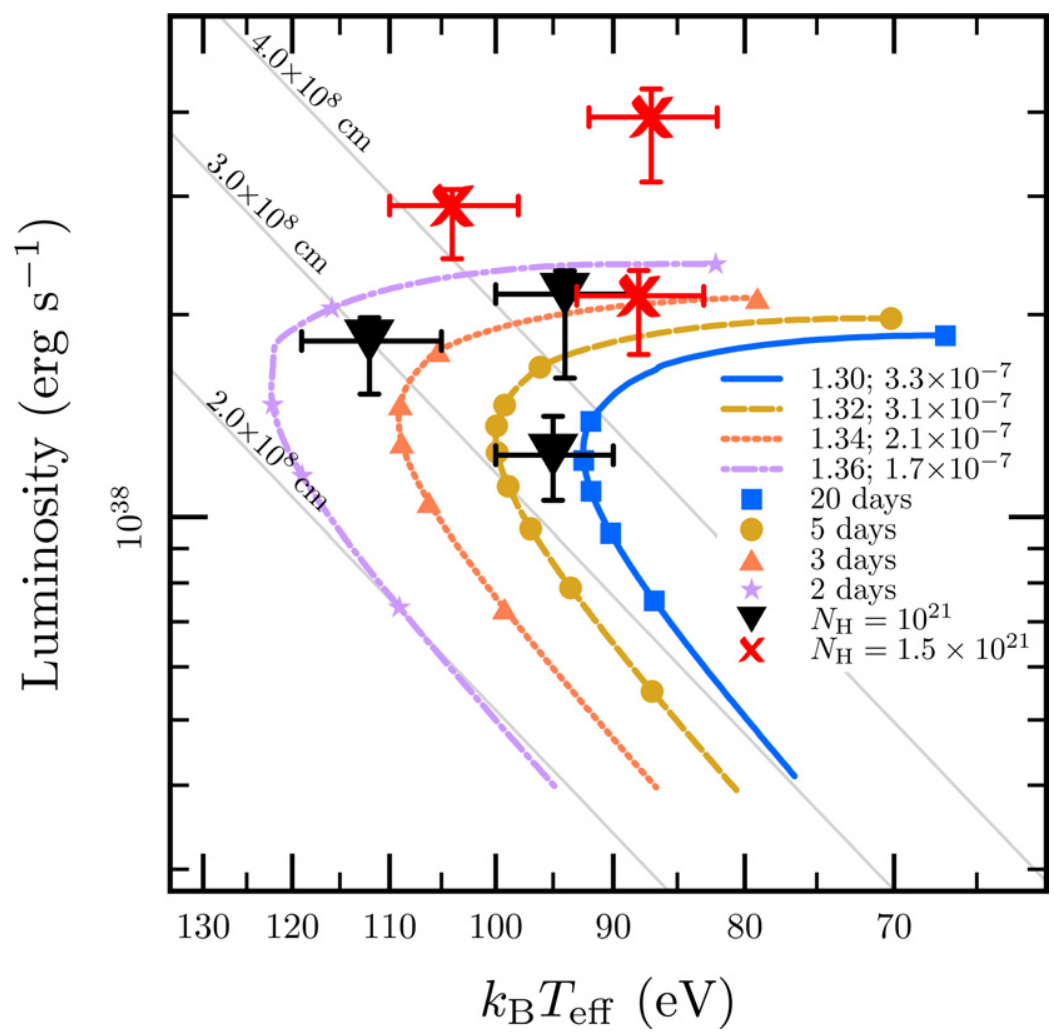

Figure 5. Comparison of observations vs. theoretical models on the Luminosity (bolometric) vs. $k_{B} T_{\text {eff }}$ plane during the SS phase. Best-fit results from the Swift XRT data using an absorbed blackbody model with fixed $N_{\mathrm{H}}=1.0 \times 10^{21} \mathrm{~cm}^{-2}$ and $N_{\mathrm{H}}=1.5 \times 10^{21} \mathrm{~cm}^{-2}$ are shown in black upside down triangles and red crosses, respectively, with the top-right point from the early stage (5-8 days after optical peak), the middle-left point from the middle stage (8-11 days after optical peak), and the lower-right point from the late stage (11-17 days after optical peak). Note that $L_{X}(0.3-1 \mathrm{keV})$ in Table 4 are converted to bolometric luminosity assuming blackbody radiation with best-fit temperatures. Evolutionary tracks for the four MESA models with $t_{\text {recur }} \approx 1$ yr are shown in thick colored lines with markers; all the tracks evolve from higher luminosity to lower luminosity. Different lines indicate different WD mass and $M$ combinations (indicated in the legend in units of $M_{\odot}$ and $M_{\odot} \mathrm{yr}^{-1}$ ), and the markers separate periods of equal time which are also indicated in the legend. More massive stars yield shorter SSS phases due to the smaller envelope masses as well as the more vigorous burning (Wolf et al. 2013). Also shown in gray are lines of constant radius.

(A color version of this figure is available in the online journal.)

and $1.7 \times 10^{-7} M_{\odot} \mathrm{yr}^{-1}$. These models retained $\approx 30 \%$ of the accreted material through the outburst, yielding effective accretion rates onto the helium layer of $\dot{M}_{\mathrm{WD}} \approx 9 \times 10^{-8}, 9 \times 10^{-8}$, and $6 \times 10^{-8} M_{\odot} \mathrm{yr}^{-1}$. Assuming this matter ultimately stays on the WD and that the effective accretion rate remains constant, these models would evolve to $M=1.37 M_{\odot}$ within $5 \times 10^{5} \mathrm{yr}$.

We next studied the location of these high mass WDs in the HR diagram during the SSS phase, all of which enter the SSS phase after only about 10-20 days from the onset of the TNR. As listed in Table 4, the measured $T_{\text {eff }}$ from the two absorbed blackbody models are consistent with each other within uncertainties, though there is a modest discrepancy in the blackbody luminosities. As shown in Figure 5, all theoretical tracks are consistent with the measured $T_{\text {eff }}$ of RX J0045.4+4154, with either $N_{\mathrm{H}}=1.0 \times 10^{21} \mathrm{~cm}^{-2}$ or $N_{\mathrm{H}}=1.5 \times 10^{21} \mathrm{~cm}^{-2}$. However, the WDs have different rates of evolution in the HR diagram depending on their mass, as denoted by the solid points that denote the elapsed time between each point. For example, the $1.34 M_{\odot}$ WD (orange dotted line and triangles) evolves through the SSS phase in about 15 days, whereas the $1.36 M_{\odot}$ WD is in a SSS phase for only 8 days. Clearly, the $1.30 M_{\odot}$ WD evolves far too slowly. So, this comparison again points to WD masses of $1.30<M / M_{\odot}<1.36$ in our super Eddington wind model, with mass loss due to Roche lobe overflow indicating more massive WDs (Wolf et al. 2013). The turnoff times could be shorter if there was substantial mixing of elements heavier than hydrogen (e.g., He, C, O) into the burning layer during the TNR (Sala \& Hernanz 2005), pointing to WD masses at the lower end, i.e., $M=1.30 M_{\odot}$.

\section{CONCLUSION}

As an RN, RX J0045.4+4154 has many remarkable features, with the shortest recurrence time of $1 \mathrm{yr}$, rapid turn-on and turn-off of the stable burning SSS phase, and the highest peak effective temperature during the SSS (100-110 eV). We showed here that these are all remarkably consistent with theoretical models of hydrogen thermonuclear flashes on a WD with a mass in the range of $1.30<M / M_{\odot}<1.36$. Securely identifying such a massive WD is key to our understanding of the larger problem of deciding how some massive WDs lead to explosions as Type Ia $\mathrm{SNe}$ (if the core is carbon rich) or undergo an accretion-induced collapse (when the core is composed of $\mathrm{O} / \mathrm{Ne}$ ). At the accretion rate inferred from our theoretical calculations, and assuming (from the duration of the supersoft phase) that about $30 \%$ of the accreted material stays on the WD, it will take less than a million years for the core density of the accreting WD to reach values adequate for either an unstable carbon ignition or onset of electron capture (if an $\mathrm{O} / \mathrm{Ne}$ core). This assumes that mass loss in the inevitable intervening unstable helium flashes is negligible (Kato \& Hachisu 2004). 
This remarkable system will certainly undergo additional outbursts, and we hope that the next one (likely in 2014 November-December) will be studied in even more detail, especially in the X-ray region where spectra can certainly reveal much more about the WD mass and surface composition. More detailed theoretical modeling is indubitably justified, including simulations that resolve the state of the accumulating helium layer.

This work was supported by the National Science Foundation under grants PHY 11-25915, AST 11-09174, and AST 12-05574. Most of the MESA simulations for this work were made possible by the Triton Resource. The Triton Resource is a high-performance research computing system operated by the San Diego Supercomputer Center at UC San Diego. This research used resources of the National Energy Research Scientific Computing Center, which is supported by the Office of Science of the U.S. Department of Energy under Contract No. DE-AC02-05CH11231. A.K.H.K. is supported by the National Science Council of the Republic of China (Taiwan) through grant NSC101-2119-M-008-007-MY3. M.M.K. acknowledges generous support from the Hubble Fellowship and CarnegiePrinceton Fellowship. We are grateful to the Swift Team for the superb timely scheduling of the observations and providing data and analysis tools, and to Bill Paxton for his development of MESA.

Facilities: PO:1.2m (PTF), PO:1.5m, Keck:I (DEIMOS, LRIS), Swift (XRT, UVOT)

\section{REFERENCES}

Arp, H. C. 1956, AJ, 61, 15

Barsukova, E., Fabrika, S., Hornoch, K., et al. 2011, ATel, 3725, 1 Boulade, O., Charlot, X., Abbon, P., et al. 2003, Proc. SPIE, 4841, 72 Burrows, D. N., Hill, J. E., Nousek, J. A., et al. 2005, SSRv, 120, 165 Cao, Y., Kasliwal, M. M., Neill, J. D., et al. 2012, ApJ, 752, 133

Cassisi, S., Iben, I. J., \& Tornambe, A. 1998, ApJ, 496, 376

Cenko, S. B., Fox, D. B., Moon, D.-S., et al. 2006, PASP, 118, 1396

Darnley, M. J., Bode, M. F., Kerins, E., et al. 2004, MNRAS, 353, 571

Darnley, M. J., Williams, S. C., Bode, M. F., et al. 2014, A\&A, 563, L9

della Valle, M., \& Livio, M. 1995, ApJ, 452, 704

Denissenkov, P. A., Herwig, F., Bildsten, L., \& Paxton, B. 2013, ApJ, 762, 8

Di Stefano, R. 2010, ApJ, 719, 474

Dolphin, A. E. 2000, PASP, 112, 1383

Evans, P. A., Beardmore, A. P., Page, K. L., et al. 2007, A\&A, 469, 379

Faber, S. M., Phillips, A. C., Kibrick, R. I., et al. 2003, Proc. SPIE, 4841, 1657

Gehrels, N., Chincarini, G., Giommi, P., et al. 2004, ApJ, 611, 1005

Henze, M., Ness, J.-U., Bode, M. F., Darnley, M. J., \& Williams, S. C. 2013a, ATel, 5627, 1

Henze, M., Ness, J.-U., Bode, M. F., et al. 2013b, ATel, 5633, 1
Henze, M., Ness, J.-U., Darnley, M. J., et al. 2014b, A\&A, 563, L8

Henze, M., Pietsch, W., Haberl, F., et al. 2010, A\&A, 523, A89

Henze, M., Pietsch, W., Haberl, F., et al. 2014a, A\&A, 563, A2

Hubble, E. P. 1929, ApJ, 69, 103

Kasliwal, M. M., Cenko, S. B., Kulkarni, S. R., et al. 2011, ApJ, 735, 94

Kato, M., \& Hachisu, I. 2003, ApJL, 587, L39

Kato, M., \& Hachisu, I. 2004, ApJL, 613, L129

Korotkiy, S., \& Elenin, L. 2010, Central Bureau for Astronomical Telegrams, http://www.cbat.eps.harvard.edu/unconf/followups/J00452885+ 4154094.html

Law, N. M., Kulkarni, S. R., Dekany, R. G., et al. 2009, PASP, 121, 1395

Massey, P., Olsen, K. A. G., Hodge, P. W., et al. 2006, AJ, 131, 2478

Miller, J. S., \& Stone, R. P. S. 1993, Lick Obs. Tech. Rep., 66 (Santa Cruz, NM: Lick Obs.)

Nishiyama, K., \& Kabashima, F. 2008, Central Bureau for Astronomical Telegrams, http://www.cbat.eps.harvard.edu/iau/CBAT_M31.html\#2008-12a

Nishiyama, K., \& Kabashima, F. 2012, Central Bureau for Astronomical Telegrams, http://www.cbat.eps.harvard.edu/unconf/followups/J00452884+ 4154095.html

Ofek, E. O., Laher, R., Law, N., et al. 2012, PASP, 124, 62

Oke, J. B., Cohen, J. G., Carr, M., et al. 1995, PASP, 107, 375

Orio, M., Nelson, T., Bianchini, A., Di Mille, F., \& Harbeck, D. 2010, ApJ, 717,739

Osterbrock, D. E., \& Ferland, G. J. 2006, Astrophysics of Gaseous Nebulae and Active Galactic Nuclei (Sausalito, CA: Univ. Science Books)

Paxton, B., Bildsten, L., Dotter, A., et al. 2011, ApJS, 192, 3

Paxton, B., Cantiello, M., Arras, P., et al. 2013, ApJS, 208, 4

Piersanti, L., Cassisi, S., Iben, I. J., \& Tornambe, A. 1999, ApJL, 521, L59

Pietsch, W., Fliri, J., Freyberg, M. J., et al. 2005, A\&A, 442, 879

Poole, T. S., Breeveld, A. A., Page, M. J., et al. 2008, MNRAS, 383, 627

Predehl, P., \& Schmitt, J. H. M. M. 1995, A\&A, 293, 889

Prialnik, D., \& Kovetz, A. 1995, ApJ, 445, 789

Rauch, T., Ringat, E., \& Werner, K. 2010, arXiv:1011.3628

Roming, P. W. A., Kennedy, T. E., Mason, K. O., et al. 2005, SSRv, 120, 95

Sala, G., \& Hernanz, M. 2005, A\&A, 439, 1061

Schaefer, B. E. 2010, ApJS, 187, 275

Schlafly, E. F., \& Finkbeiner, D. P. 2011, ApJ, 737, 103

Shafter, A. W., Curtin, C., Pritchet, C. J., Bode, M. F., \& Darnley, M. J. 2013, arXiv: 1307.2296

Shafter, A. W., Darnley, M. J., Hornoch, K., et al. 2011, ApJ, 734, 12

Shafter, A. W., Hornoch, K., Ciardullo, J. V. R., Darnley, M. J., \& Bode, M. F. 2012, ATel, 4503, 1

Starrfield, S., Sparks, W. M., \& Shaviv, G. 1988, ApJL, 325, L35

Starrfield, S., Sparks, W. M., \& Truran, J. W. 1974, ApJS, 28, 247

Supper, R., Hasinger, G., Lewin, W. H. G., et al. 2001, A\&A, 373, 63

Tang, S., Cao, Y., \& Kasliwal, M. M. 2013, ATel, 5607, 1

Vilardell, F., Ribas, I., Jordi, C., Fitzpatrick, E. L., \& Guinan, E. F. 2010, A\&A, 509, A70

White, N. E., Giommi, P., Heise, J., Angelini, L., \& Fantasia, S. 1995, ApJL, 445, L125

Williams, B. F., Garcia, M. R., Kong, A. K. H., et al. 2004, ApJ, 609, 735

Williams, R. E. 1992, AJ, 104, 725

Williams, S. C., Darnley, M. J., Bode, M. F., \& Shafter, A. W. 2013, ATel, 5611,1

Wolf, W. M., Bildsten, L., Brooks, J., \& Paxton, B. 2013, ApJ, 777, 136

Yamanaka, M., Uemura, M., Kawabata, K. S., et al. 2010, PASJ, 62, L37

Yaron, O., Prialnik, D., Shara, M. M., \& Kovetz, A. 2005, ApJ, 623, 398 